\title{
LO REALMENTE IMPORTANTE NO ES VIVIR, SINO VIVIR BIEN. UNA DISCUSIÓN SOBRE EUTANASIA, AUTONOMÍA Y AUTORRESPETO
}

\author{
Pablo Aguayo Westwood ${ }^{1}$
}

Resumen: Este artículo tiene por objetivo introducir la noción de “autorrespeto" en el marco de la discusión sobre la eutanasia. Para ello muestra brevemente, en primer lugar, dos rasgos que caracterizan gran parte de los debates morales contemporáneos sobre temas de ética aplicada, rasgos que permitirán explicar, con mayor claridad, la necesidad de ampliar las razones que permitan a las personas estar en condiciones de adelantar su muerte. En segundo término, se expone una de las primeras reflexiones de Platón acerca de la diferencia entre el mero hecho de estar vivos (to zen) y el vivir bien (to eu zen), y cuyos argumentos podemos trasladar en parte al debate sobre la eutanasia. Luego de discutir la idea de "persona", el artículo aborda finalmente la noción de "autorrespeto" como criterio para considerar la legitimidad moral de poner anticipadamente fin a nuestra vida.

Palabras clave: eutanasia, suicidio asistido, autonomía personal, autorrespeto

The really important thing is not to live, but to live well. A discussion about euthanasia, autonomy and self-respect

\begin{abstract}
This paper aims to introduce the notion of self-respect in the context of the discussion on euthanasia. First, I briefly show two features that characterize much of the contemporary moral debates on issues of applied ethics, features that will allow me to explain more clearly the discussion about euthanasia. Looking back at the history of Philosophy, in the second part, I will show how Plato offered one of the first reflections on the difference between the mere fact of being alive (to zen) and living well (to eu zen), and whose arguments we can use to discuss euthanasia. Third, I will examine what we understand by a person and how a particular comprehension of this notion will allow me to elaborate arguments in favour of euthanasia based on the idea of self-respect.
\end{abstract}

Keywords: euthanasia, assisted suicide, personal autonomy, self-respect

O realmente importante não é viver, mas sim viver bem. Uma discussáo sobre eutanásia, autonomia e auto-respeito

Resumo: Este artigo tem por objetivo introduzir a noção de "auto-respeito" no contexto da discussão sobre eutanásia. Para isto, mostra brevemente, em primeiro lugar, dois aspectos que caracterizam grande parte dos debates morais contemporâneos sobre temas de ética aplicada, aspectos estes que permitirâo explicar, com maior clareza, a necessidade de ampliar as razóes que permitam às pessoas estarem em condições de adiantar sua morte. Em segundo lugar, expóe-se uma das primeiras reflexões de Platão acerca da diferença entre o mero fato de estar vivos (to zen) e o viver bem (to eu zen), e cujos argumentos podemos transferir em parte ao debate sobre a eutanásia. Depois de discutir a ideia de "pessoa", o artigo aborda finalmente a noção de "auto-respeito" como critério para considerar a legitimidade moral de por antecipadamente fim à nossa vida.

Palavras chave: eutanásia, suicidio assistido, autonomia pessoal, auto-respeito

\footnotetext{
${ }^{1}$ Facultad de Derecho, Universidad de Chile, Chile

Correspondencia: paguayo@derecho.uchile.cl
} 


\section{Introducción}

Parte importante del debate sobre la legalidad de la eutanasia se ha centrado en los criterios exigibles para que una persona pueda solicitarla, así como quién debe realizarla(1). Estos criterios varían dependiendo de la legislación de cada país(2), así como también del contexto social, político y valorativo en el que se discute. Asimismo, una extensa literatura ha intentado precisar la diferencia entre las diferentes formas de eutanasia y los casos límites en los que esta se acerca al suicidio asistido $(3,4)$. Todas estas discusiones parten de algunos presupuestos filosóficos básicos, expresados en nociones como "libertad", "autonomía", "persona" y "dignidad", los que se complementan con elementos de la praxis médica.

En lo que sigue, quiero mostrar la pertinencia de introducir la noción de "autorrespeto" en el marco de la discusión acerca de las razones que podrían ofrecerse para solicitar la eutanasia. Para lo anterior, discutiré brevemente dos rasgos que caracterizan parte importante de los debates morales contemporáneos sobre temas de ética aplicada, rasgos que me permitirán explicar con mayor claridad la discusión sobre la eutanasia. Volviendo la mirada a la historia de la filosofía, posteriormente mostraré cómo Platón ofreció una de las primeras reflexiones sobre la diferencia entre el mero hecho de estar vivos y el vivir bien, y cuyos argumentos podemos trasladar a este debate. Finalmente discuto lo que entendemos por persona y cómo una determinada comprensión de esta noción permite elaborar argumentos a favor de solicitar anticipadamente nuestra muerte.

\section{Características de los debates morales contem- poráneos}

En primer lugar, el debate acerca de la moralidad de las diferentes formas de eutanasia, así como también otros debates, como el aborto o la maternidad subrogada, tiene al menos dos rasgos que lo caracterizan y que llevan a cierta perplejidad(5). El primero es la inconmensurabilidad conceptual de las argumentaciones rivales. Este rasgo implica que cada uno de los argumentos que se presenta al debate es lógicamente válido y las conclusiones se siguen de las premisas. Pero lo que sucede es que las premisas que sirven de base a los argu- mentos son tales que no tenemos ninguna manera racional de sopesar sus pretensiones cuando las comparamos entre sí. Lo anterior se debe a que en cada premisa se emplea algún concepto normativo o valorativo, por ejemplo, los conceptos de "vida", "muerte", "libertad", "obligación", cuya comprensión por parte de los interlocutores es completamente diferente. Lo anterior hace que, aunque parece que discutieran sobre lo mismo, en realidad existe tal diversidad de significados de los conceptos centrales, que hace difícil un entendimiento recíproco.

El segundo de estos rasgos es la pretensión de racionalidad e impersonalidad de las argumentaciones rivales. Considérese por un segundo que estamos en un acalorado debate sobre si debemos permitir el aborto a menores de 16 años sin consentimiento de sus padres o tutores. Imaginemos que, en medio del debate, alguien sostiene que las autoridades deberían tomar tal o cual decisión. Frente a tal aseveración uno podría preguntar por qué deberían hacer tal o cual cosa. Ante ello, nuestro interlocutor tiene dos opciones: o bien decir que deberían hacer eso "porque él o ella lo cree", o intentar mostrar que su juicio se desprende lógicamente de una determinada concepción de la justicia, del deber o de la vida humana. Al hacer lo segundo, nuestro interlocutor está intentando apelar a criterios impersonales, los cuales se suponen independientes de la relación entre el hablante y el oyente, pero ¿es esto posible? Esta pregunta quedará abierta por ahora y la retomaré en la parte final del trabajo.

A continuación, revisaré algunos pasajes de $E l$ Critón de Platón para mostrar cómo los argumentos de Sócrates para beber la cicuta engarzan con la reflexión sobre la vida buena, así como con la necesidad de acabar con ella cuando esta no nos permite ser y hacer lo que somos y queremos ser.

\section{No es lo mismo vivir que vivir bien}

El título de este artículo alude directamente a una parte central del diálogo de Platón titulado El Critón(6). En dicho diálogo, Platón detalla los argumentos que Sócrates esgrime para beber la cicuta y no aceptar la oferta de escapar de prisión. La relevancia de la argumentación de Sócrates es que pone en discusión nuestra comprensión del 
valor de la vida y lo que significa sostener y proteger ese valor.

La discusión entre Sócrates y Critón en el diálogo homónimo está centrada en las razones que podrían justificar que Sócrates escape de prisión y no acate la sentencia que lo obliga a beber la cicuta. El contenido de la discusión es relevante, ya que los argumentos de Critón para convencerlo de que escape responden en gran medida a razones emocionales centradas en una cierta concepción de la vida humana, en particular de la vida virtuosa, según ciertos cánones de la época. Asimismo, los argumentos de Critón reflejan más que un interés por la vida de Sócrates y su decisión autónoma, un interés por los afectados de su decisión. De modo sintético, se pueden resumir en los siguientes puntos:

Argumento de la amistad y el afecto: Si Sócrates permite que le ejecuten, Critón y sus amigos se verán privados de un amigo irremplazable.

Argumento de la traición: Si Sócrates permite que le ejecuten, se habrá traicionado a sí mismo, ya que habrá hecho lo que sus enemigos querían que hiciera (entendiendo "traición" como hacer algo que coincide con los propósitos del enemigo).

Argumento de la cobardía: Si Sócrates permite que le ejecuten, se revelará a sí mismo como carente de las cualidades de un hombre bueno y valiente. El argumento se puede extender a la situación de sus amigos.

Frente a los anteriores argumentos, Sócrates presenta dos principios normativos desde los cuales pretende justificar su decisión. Estos principios son:

No es el vivir (to zen) lo que por sobre todo debe ser valorado, sino el vivir bien (to eu zen).

El vivir bien (to eu zen) es lo mismo que vivir bella (kalos) y justamente (dikaios)(6)2.

En su diálogo con Critón, Sócrates se esfuerza

${ }^{2}$ En el contexto de la moralidad popular griega de la época, el vocablo dikaios aplicado a nuestra vida expresa un rango de valoraciones que podemos asimilar a "honesta", "razonable" y "justificada". Es este último sentido el que se acerca al razonamiento que quiero desarrollar a continuación. Sigo aquí a Kenneth Dover en Greek popular morality in the time of Plato and Aristotle (7, p. 185). en mostrar las nefastas consecuencias que tendría para él vivir fuera de Atenas. Téngase presente que, para Sócrates, esa experiencia no equivale a la situación de, por ejemplo, una persona que hoy tenga que exiliarse por razones políticas. Recuérdese que, para los griegos, todos sus vecinos eran unos bárbaros, es decir, pueblos cuya lengua sonaba a sus oídos como un balbuceo incompresible que haría imposible la comunicación con ellos y, por tanto, no les sería posible llevar adelante su vida como filósofo. Dicho lo anterior, resulta inevitable reconocer en el argumento de Sócrates una clara distinción entre tener una vida y el hecho de que esta sea llevada delante de manera buena, noble y justificada ${ }^{3}$.

\section{Eutanasia y el valor de la vida de las personas}

Como sostuve al inicio, nuestra postura respecto del significado moral de la eutanasia está inevitablemente ligada a la manera como comprendemos el valor de la vida y lo que significa proteger ese valor. En este sentido, lo primero que debo aclarar es que, cuando hablamos acerca del valor de la vida, podemos defender ese valor en abstracto o bien como el valor de la vida de una persona en particular, o incluso, si se quiere, en términos generales, aunque no abstractos, como el valor de la vida de las personas.

La anterior distinción es fundamental para el argumento que quiero presentar aquí a favor tanto de la eutanasia voluntaria pasiva, es decir la renuncia deliberada a medidas paliativas destinadas al mantenimiento de la vida cuando las condiciones de esta última resultan tanto física como psicológicamente insoportables; como de la eutanasia voluntaria activa indirecta, es decir, la aplicación de medios paliativos que, como consecuencia, podrían acelerar la muerte (doctrina del doble efecto) y, por sobre todo, de la eutanasia voluntaria activa directa y el suicidio asistido a petición expresa de la persona ${ }^{4}$.

Mi argumento a favor de estas tres formas de ade-

\footnotetext{
${ }^{3}$ Cabe destacar aquí que, si bien Sócrates no se encuentra aquejado por una enfermedad terminal, ni tampoco padece dolores físicos imposibles de paliar, él considera que una forma de vida diferente a la que ha llevado no merece la pena.

${ }^{4}$ Hace ya más de cuatro décadas, James Rachel, en Active and Passive Euthanasia, puso en discusión la tesis según la cual la eutanasia pasiva era moralmente menos condenable que la eutanasia activa(9).
} 
lantar nuestra muerte descansa principalmente en dos ideas. La primera es la autonomía personal como límite moral infranqueable al momento de tomar decisiones que afectan el final de nuestras vidas y, en segundo lugar, el autorrespeto como condición necesaria para poder llevar adelante una vida significativa. Si bien la primera idea ya ha sido explorada por la literatura, la ventaja que tiene introducir la noción de "autorrespeto" es novedosa y es lo que desarrollaré al final de este trabajo 5 .

Sobre la noción de "persona", no pretendo ofrecer aquí una genealogía o una breve historia de ella; solo he de señalar que me parece completamente equivocado, o al menos incorrecto, pensar que existe una noción unívoca. No solo su significado varía si hablamos de persona moral o de persona jurídica, sino que cada tradición jurídica y moral ha defendido una concepción diferente. Contemporáneamente, John Harris ha sostenido que el rasgo central que define a una persona es "su capacidad para valorar su propia existencia” (8). Para Harris, la idea de que una persona sea entendida como una criatura capaz de valorar su propia existencia le permite hacer verosímil la explicación de la naturaleza del mal que se le produce a alguien cuando se le priva de su vida. Cada uno de nosotros siente un mal cuando se nos priva de algo que valoramos. De hecho, la misma noción de mal ha de entenderse en nuestra tradición judeo-cristina como carencia, como no-ser. En este sentido, la muerte sería el mal radical, la ausencia absoluta de ser y, por tanto, una condición en la cual resultaría imposible valorar cualquier otro tipo de bienes.

Asimismo, entender a las personas como criaturas

\footnotetext{
${ }^{5}$ La principal diferencia entre apelar a la autonomía y apelar a las condiciones que definen el autorrespeto como criterio a considerar para solicitar la eutanasia, se basa en que la primera es una noción que descansa en una particular concepción de la moral, de tradición kantiana, que se ve reflejada en el ordenamiento jurídico constitucionalmente, mediante la idea de dignidad, y civilmente, por medio de la idea de acto voluntario-, mientras que la segunda responde a una autocomprensión del sujeto en lo que concierne al valor de su propia vida, así como a la suficiencia de sus medios para llevarla adelante. En este sentido, una persona podría tener todas las condiciones que lo hacen un agente legalmente capaz y, pese a ello, considerar que su actual condición física o psicológica hace imposible llevar su vida adelante. Esta segunda vía de argumentación no ha sido explorada por la literatura, por lo que en parte justifica su tratamiento en este artículo.
}

capaces de valorar su propia existencia serviría de fundamento a la idea de que a aquellas que no quieren vivir y que no valoran su existencia, no se les estaría haciendo un mal cuando se les concede el derecho a morir ${ }^{6}$. En este mismo sentido, sería difícil afirmar la importancia que el propio valor de la vida tiene cuando, al mismo tiempo, se niegue el derecho autónomo de terminar con ella. De todos modos, hay que aclarar que quienes expresamente clara y conscientemente su deseo de morir porque no valoran su vida, o porque esta se les hace "invivible", no dejan de ser personas para nosotros. De hecho, es en virtud de este reconocimiento, y en particular del reconocimiento al valor de su autonomía en sentido moral, que debemos considerar su capacidad para determinar el curso de su propia vida.

Insisto en que el argumento anterior descansa en que lo que estimamos no es el valor de la vida en general, sino el valor de la vida de esta persona en particular, el valor de su autonomía. Teniendo entonces a la autonomía como un valor y límite infranqueable de las decisiones personales, las formas de eutanasia voluntarias no constituirian un daño moral.

Así, entonces, si lo sagrado es la vida de las personas, no estaríamos cometiendo una falta moral en los casos de eutanasia anteriormente señalados, incluso en el caso de eutanasia activa. Esta tesis, que en principio parece tan radical y que pareciera atentar contra los valores cristianos, ha sido defendida por teólogos como el sacerdote Hans Küng, en su obra Morir con dignidad. Un alegato a favor de la responsabilidad. Para Kung, quienes se oponen a todo tipo de eutanasia apelando a que la vida humana es un don de Dios cuyo rechazo equivaldría a una "transgresión de una ley divina" (10:56) sostienen argumentos en los que solo se ofrece "una imagen distorsionada de Dios, basada en textos unilateralmente seleccionados de la Biblia (...) donde nada hay del Dios padre de los débiles, dolientes y extraviados, del Dios solida-

\footnotetext{
${ }^{6}$ Aquí resulta relevante la distinción entre "asistencia al suicidio" y "eutanasia activa”, encontrándose esta última con una serie de exigencias, tales como la presencia de otros facultativos y que la persona se encuentre en un estado de enfermedad terminal con dolores que no han podido ser aplacados. Por otra parte, el bien jurídico que protege el art. 393 del Código Penal Chileno está pensado para un bien al que la persona que solicita la eutanasia ya ha renunciado voluntariamente.
} 
rio de la Alianza"(10:56-57). Cabe destacar que, a pesar de que la opinión de Küng es favorable a algunas formas de eutanasia, él está en contra de Peter Singer, según el cual una persona, por las deficiencias de su ancianidad o por su definitiva inconciencia, deja de ser persona. La posición de Küng es justo la contraria. Sostiene que "porque la persona humana es persona y lo sigue siendo siempre, también, aunque esté mortalmente enferma o moribunda, tiene derecho a una muerte y una despedida digna de humanos, un derecho que con tanta dependencia indefinida de aparatos y de medicamentos probablemente se desvirtúe durante el proceso que puede durar meses e incluso años hasta alcanzar una mera existencia vegetativa"(10:58).

Pero Hans Küng no es el único teólogo que ha establecido ciertas condiciones para una legítima eutanasia. El teólogo protestante holandés Harry M. Kuitert ha presentado las siguientes condiciones: (a) que la solicitud ha de hacerla el propio enfermo, la que debe ser meditada y sostenida en el tiempo; (b) el estado vital insoportable y doloroso ha de justificar esa solicitud; (c) la eutanasia está reservada solo al médico; (d) el médico debe consular previamente con un colega sobre la seriedad de la solicitud, el acierto en la apreciación del estado del paciente y la mejor forma del procedimiento; (e) el médico deberá redactar un informe sobre estas condiciones que, según la propia ley holandesa, debe ser enviado al Ministerio Público quien se pronunciará sobre la misma, evitando así cualquier tipo de proceso penal en contra el médico o del propio paciente(11).

El propósito de mostrar la opinión de estos dos teólogos es remarcar la tesis de la inconmensurabilidad conceptual y la pretensión de racionalidad impersonal. Lo anterior porque parte del discurso moral en contra de la eutanasia descansa en lo que, con Rawls, podemos llamar un rechazo, tanto al pluralismo razonable, como a la necesidad de que en una sociedad democrática pueden convivir varias y variadas doctrinas comprehensivas(12). Parte importante de quienes rechazan la eutanasia lo hacen por razones asentadas en la creencia de que su propia doctrina comprehensiva es la única defendible, o en que sus argumentos se levantan con independencia de su propio entendimiento de lo que es una vida buena o digna $(4,13,14)^{7}$. Pero, como es posible observar, las personas tenemos diferentes entendimientos no solo de la vida, sino de las condiciones mínimas para que esta merezca ser vivida. Asimismo, no parece claro por qué deberíamos aceptar acríticamente un orden léxico de los valores a partir del cual mis propias razones para pedir terminar con mi vida de manera anticipada sean menos valiosas que otras, sobre todo cuando estas últimas podrían estar en contra del principio de autonomía y al límite del de nomaleficencia(15).

\section{Eutanasia y autorrespeto}

Llegados a este punto, quisiera presentar una última vía de argumentación a favor de las formas de eutanasia enunciadas anteriormente ${ }^{8}$. La razón para introducirla descansa en que, si bien el argumento de la autonomía es moralmente potente para resguardar la libertad de elección de las personas, puede resultar insuficiente al momento de valorar las condiciones que, en una serie casos, ponen en duda los méritos de quien solicita terminar su vida de manera anticipada. De hecho, una persona podría contar con todas las garantías morales y jurídicas asociadas a su autonomía personal y, pese a ello, estar privada de poder adelantar su muerte dado el marco legal vigente, marco que recoge una moralidad específica.

En una parte importante de los argumentos a favor de la eutanasia se establece la necesidad de que la persona se encuentre en una situación tal que merezca acceder a una muerte anticipada, es decir, el derecho a la eutanasia se encuentra, de algún modo, limitado a una serie de condiciones establecidas por la legislación vigente que responde a una moralidad particular. Esta limitación descansa, entre otras razones, en el miedo a una posible "pendiente resbaladiza" que nos lleve a traspasar el marco moral en el que se sustenta la eutanasia

\footnotetext{
${ }^{7}$ Esta es una de las razones por las que he evitado usar la noción de "dignidad" y he preferido discutir hasta qué punto la idea de autorrespeto podría satisfacer mejor las condiciones de pluralidad axiológica, propia de nuestras sociedades democráticas. En principio, la idea de autorrespeto no descansa en una determinada comprensión de la vida buena, sino más bien en una serie de indicios que permiten valorar hasta qué punto un determinado plan de vida puede llevarse a cabo.

${ }^{8}$ Este argumento también puede, en principio, trasladarse al suicidio asistido.
} 
y hacer de ella un instrumento que responda a lógicas de eficiencia sanitaria, o incluso de paso a eutanasias de vidas sin valor. Pero dejando de lados esas razones, las principales limitaciones a la eutanasia van asociadas a condiciones de sufrimiento físico o psíquico insoportable, así como al carácter irreversible de una enfermedad(16). Pero, ¿qué sucede en aquellos casos en que estas condiciones no se dan pero, pese a ello, la persona desea poner fin anticipado a su vida por encontrar que esta no tiene valor? ¿Es posible apelar a otra noción más allá de la autonomía personal?

$\mathrm{Al}$ inicio de este artículo mostré brevemente cómo Sócrates había marcado con claridad la distinción entre vivir y vivir bien. El vivir bien claramente está asociado a tener un proyecto de vida que no solo yo encuentre valioso, sino que sea reconocido por quienes yo aprecio. Asimismo, es de vital importancia la confianza que cada uno debe tener en que sus medios son suficientes para llevar adelante el plan de vida que se ha trazado. Son estas condiciones las que definen el autorrespeto de las personas y que creo podrían aportar a la discusión sobre la eutanasia, como veré a continuación(17:440).

Resulta difícil no considerar que el autorrespeto es una condición fundamental para que las personas podamos llevar adelante nuestros planes de vida $y$, de ese modo, considerar que esta vida vale la pena. La relevancia moral y psicológica del autorrespeto nos lleva a pensar que sin él nada parece valioso de llevarse a cabo, o si algunas cosas tienen valor para nosotros, nos falta la voluntad para luchar por ellas. De hecho, el autorrespeto es seguramente una de las dimensiones morales más significativas de la vida humana. La posibilidad de valorarse y respetarse es algo de vital importancia para llevar adelante una vida significativa y satisfactoria. Por lo anterior, es posible sostener que una persona que posee autorrespeto tiene un conjunto de ideas, creencias y valores a través de las cuales vive y organiza su vida; se reconoce a sí misma como poseyendo valor moral, lo que le lleva a sentir vergüenza por sus fracasos y un legítimo orgullo por sus éxitos. Por otra parte, quienes carecen de autorrespeto - $\mathrm{o}$ tienen un frágil y dañado respeto de sí- están en principio condenados a llevar una vida difícil e incluso deformada, con pocas posibilidades de autorrealización y felicidad(18).

Dada la relevancia del autorrespeto para el desarrollo de la vida de las personas, considero que esta dimensión de nuestra vida moral y psicológica también debe tenerse en cuenta como criterio en la discusión de los argumentos válidos al momento de decidir poner fin a nuestras vidas de manera anticipada. De este modo, una persona que sostiene que su plan de vida no puede llevarse adelante porque sus actuales condiciones físicas y/o psíquicas se lo impiden —es decir, porque no dispone objetivamente de los medios para realizarlo-, no debería ser tratada de modo diferente en comparación con aquellas que ofrecen argumentos aceptados por varias legislaciones vigentes $(16,17)$. Pienso aquí en personas que no se encuentran necesariamente en una fase terminal de una enfermedad irreversible, ni tampoco padecen dolores o sufrimientos insoportables, pero sí se encuentran en una situación tal que no cuentan con las condiciones que hacen posible tener un debido respeto de sí. Ejemplos de lo anterior serían hombres y mujeres cuyo trabajo, oficio y forma de vida descansa en una cierta perfección de su cuerpo (como deportistas de elite y artistas profesionales), o casos más complejos, como mujeres que han sido víctimas de violación y consideran que su vida ya no puede ser vivida, fruto del estrés postraumático o de una profunda pérdida del deseo de vivir ${ }^{10}$.

Debo señalar aquí que no estoy presentado la falta de respeto de sí como condición suficiente para solicitar ayuda con la finalidad de adelantar nuestra muerte, sino más bien como un elemento a considerar dentro de los otros requisitos, por

\footnotetext{
9 No se debe considerar equivalentes aquí las nociones de "autorrespeto" (self-respect) y "autoestima" (self-esteem). Por ejemplo, una persona puede tener una estima de sí muy alta y pese ello considerar que no tiene una de las condiciones del autorrespeto tal como lo hemos definido, a saber, la necesaria confianza en que sus medios y capacidades son suficientes para llevar adelante su vida. Para una distinción entre ambas, véase el artículo de David Sachs: "How to Distinguish Self-Respect from Self-Esteem"(19).

${ }^{10}$ Asimismo, para estos casos la legislación del país debería tratar de igual modo el sufrimiento psicológico y el físico. Para un estudio sobre este último punto, véase Le Glaz(20). El punto central aquí es no revictimizar a las personas que, luego de un proceso reflexivo y mantenido en el tiempo, deciden adelantar su muerte. Aceptar el resultado de su reflexión permitiría no hacerles sentir culpables por estar solicitando algo "malo", ni legal ni moralmente hablando. Como he señalado, todo este proceso de toma de decisión debe estar acompańado por personales de la salud y bajo un estricto protocolo.
} 
ejemplo, quizás en reemplazo de la condición de enfermedad irreversible o de dolor físico-psicológico insoportable. Junto a la falta de respeto de sí, se deberá considerar también que este autoentendimiento personal, así como la imposibilidad de contar con los medios para llevar la vida adelante, se manifieste durante un tiempo determinado y esté acompañado de una solicitud expresa de la persona, la que debe ser meditada y sostenida en el tiempo ${ }^{11}$.

De ser introducido el autorrespeto y las condiciones que lo definen como un elemento a valorar al momento de decidir la suficiencia de las razones para adelantar la muerte, estaríamos ampliando el rango de consideraciones morales que como sociedad nos debemos y, a su vez, respondiendo a algo tan básico como el hecho de aceptar que las personas pueden tener diversas (auto)comprensiones de lo que es llevar una vida buena, correcta y justificada.

\section{Conclusiones}

En el transcurso de este trabajo he intentado mostrar las razones que hacen pertinente introducir la noción de autorrespeto en el marco de la discusión sobre la eutanasia y el suicidio asistido. Una vez identificadas las condiciones esenciales del autorrespeto, a saber: (i) que mi plan de vida tenga valor para mí y que sea reconocido por aquellos que estimo, así como (ii) la confianza en que mis medios son suficientes para llevarla adelante, expuse la necesidad de ampliar el rango de argumentos que resultan razonables para adelantar nuestra muerte en virtud de la falta de respeto de sí. Asimismo, señalé con claridad que, si bien la falta de autorrespeto no es condición suficiente para solicitar la eutanasia y la asistencia al suicidio, sí debe ponderarse junto a otras razones que pueden estar afectando a la persona. Junto con lo anterior, sostuve que la falta de respeto de sí debe ser observada y manifestada de modo permanente en el tiempo y supeditada a otras condiciones como (i) la estricta voluntad y conciencia de la persona que la solicita y (ii) el acompañamiento de personal

\footnotetext{
${ }^{11}$ Sobre la ausencia de medios necesarios para llevar adelante la vida, cabe destacar que esta debe ser absoluta y no meramente temporal. No es posible poner al mismo nivel la falta de medios económicos con la carencia absoluta de visión, o de alguna parte del cuerpo que es condición necesaria para llevar una vida que consideramos buena y justificada.
}

capacitado. Estas condiciones permitirían evitar el contrargumento de la "pendiente resbaladiza" y evitar la creencia de que solo la falta de las condiciones para tener un debido respeto de sí son suficientes para avalar moral y jurídicamente el poner fin anticipado a nuestras vidas.

Si como sociedad plural aceptamos la existencia de un rango amplio de doctrinas comprehensivas sobre la vida buena, no podemos obligar a las personas a vivir una vida que para ellas no es ni razonable ni justificada.

\section{Agradecimientos}

Este artículo forma parte del Proyecto FONDECYT Iniciación No 11170230: "Bases sociales del autorrespeto como métrica de la justicia social".

Parte de este trabajo fue originalmente expuesto en el Seminario Anual del Comité de Bioética del Hospital Pare Jofré de Valencia, España. Agradezco la cordial invitación de su Secretario Académico, profesor Lucas J. Puig Navarro. Agradezco también al par evaluador sus sugerencias y comentarios ya que me han permitido aclarar algunas distinciones y reforzar ciertos argumentos. 
Una discusión sobre eutanasia, autonomía y autorrespeto - Pablo Aguayo Westwood

\section{Referencias}

1. Keown. J. Euthanasia Examined. Ethical, Clinical and Legal Perspective. Cambridge: Cambridge University Press; 1997.

2. Esparza E. Disposición del derecho a la vida de los menores de edad: una necesaria discusión sobre eutanasia y suicidio asistido en Chile. Acta Bioethica 2019; 24(1): 25-34.

3. Harris J. The Value of Life. London: London: Routledge; 1989.

4. Finnis J. Euthanasia, Morality, and Law. Loyola of Los Angeles Law Review 1998; 31(4): 1123-1145.

5. MacIntyre A. After Virtue: A Study in Moral Theory. London: Duckworth; 1985.

6. Platón. El Critón. Santiago de Chile: Universitaria; 2006.

7. Dover K. Greek popular morality in the time of Plato and Aristotle. Oxford: Blackwell; 1974.

8. Harris J. The concept of the Person and the Value of Life. Kennedy Institute of Ethics Journal 1999; 9(4): 293-308.

9. Rachels J. Active and Passive Euthanasia. The New England Journal of Medicine 1975; 292(9):78-80.

10. Küng H. Morir con dignidad. Un alegato a favor de la responsabilidad. Madrid: Trotta; 2010.

11. De Villiers E. Euthanasia and assisted suicide: A Christian ethical perspective. Acta Theologica 2004; 22(2): 35-65.

12. Rawls J. Political Liberalism. New York: Columbia University Press; 2005.

13. Finnis J. A philosophical case against euthanasia. In Keown. J. Euthanasia Examined. Ethical, Clinical and Legal Perspective. Cambridge: Cambridge University Press; 1997: 23-35.

14. Ramsey P. The indignity of "death with dignity". Hastings Center Studies 1974; 2: 47-62.

15. Rodríguez R. Eutanasia: aspectos éticos controversiales. Revista Médica Herediana 2001; 12(1): 32-36.

16. Dees M, Vernooij-Dassen M, Dekkers W, Vissers K, Van Weel C. 'Unbearable suffering': A qualitative study on the perspectives of patients who request assistance in dying. Journal of Medical Ethics 2011; 37(12): 727-734.

17. Rawls J. A Theory of Justice. Cambridge, Mass.: The Belknap Press of Harvard University Press; 1971.

18. Aguayo P. El rol del autorrespeto en la teoría de la justicia de John Rawls. Hybris: Revista de Filosofía 2017; 8(2): 55-76.

19. Sachs D. How to Distinguish Self-Respect from Self-Esteem. Philosophy \& Public Affairs 1981; 10(4): 346-360.

20. Le Glaz A, et al. Euthanasia for Mental Suffering Reduces Stigmatization But May Lead to an Extension of This Practice Without Safeguards. The American Journal of Bioethics 2019; 19(10): 57-59.

Recibido: 23 de diciembre de 2019

Aceptado: 16 de enero de 2020 